\title{
Improving engine efficiency by extracting laser energy from hot exhaust gas
}

\author{
Y. V. Rostovtsev, ${ }^{1}$ A. B. Matsko, ${ }^{1}$ N. Nayak, ${ }^{1}$ M. S. Zubairy, ${ }^{1,2}$ and M. O. Scully ${ }^{1,3,4}$ \\ ${ }^{1}$ Department of Physics and Institute for Quantum Studies, Texas A\&M University, Texas 77843-4242, USA \\ ${ }^{2}$ Department of Electronics, Quaid-i-Azam University, Islamabad, Pakistan \\ ${ }^{3}$ Department of Electrical Engineering, Texas A\&M University, Texas 77843, USA \\ ${ }^{4}$ Max-Planck-Institut für Quantenoptik, D-85748 Garching, Germany
}

(Received 17 June 2002; published 30 May 2003)

\begin{abstract}
We show that it is possible to improve the efficiency of a classical Otto-cycle heat engine by adding a high$Q$ microwave cavity and a laser system that can extract coherent laser energy from thermally excited "exhaust" atoms. This improvement does not violate the second law of thermodynamics, i.e., we show that a combined high- $Q$ microwave cavity and a laser system does not improve the efficiency of a classical Carnotcycle heat engine.
\end{abstract}

DOI: 10.1103/PhysRevA.67.053811

PACS number(s): 42.50.-p, 05.10.Gg, 05.40.-a

\section{INTRODUCTION}

The laws of thermodynamics were formulated before the advent of quantum mechanics. In recent years, several studies have been made to examine the viability of these laws within the framework of quantum thermodynamics $[1,2]$. These studies are particularly interesting from the point of view that quantum mechanics, which initially was stimulated by thermodynamics, now provides interesting feedback to thermodynamics [3].

Previous studies in the context of quantum thermodynamics have already shown how to extract work from a single thermal reservoir via quantum negentropy [4]. Stimulated by the preceding discussion, we have recently reconsidered various aspects of the second law in light of recent developments in quantum optics such as cavity QED [5] and lasing without inversion (LWI) [6]. The possibility of obtaining LWI for a system in thermodynamic equilibrium, e.g., in a thermal Boltzmann distribution of atomic populations, has been shown [7]. We here extend the ideas of Ref. [8] to show that it is indeed possible to improve the efficiency of certain classical heat engines. In particular, we show that the efficiency of an Otto-cycle heat engine can be improved beyond the classical limit by adding a high- $Q$ microwave cavity and a laser system that is able to extract coherent laser energy from thermally excited atoms. However, this does not violate the second law of thermodynamics because the system entropy is constantly increasing. We also derive a quantum extension of the Carnot-cycle engine, along these lines, and show that such an extension cannot improve the efficiency beyond the classical Carnot limit. Thus, the validity of the second law remains intact.

\section{THE QUANTUM AFTERBURNER CONCEPT}

In this section, we consider a closed-cycle quantum heat engine and show that its performance can be improved by extracting energy via a high- $Q$ microwave cavity and a laser system. In particular, we show that a simple Otto-cycle engine, which approximates the operation of the internal combustion gasoline engine and other similar devices, can be improved if a simple laser scheme enabling lasing from the hot exhaust gas atoms is included. The emitted laser radiation partially replaces the usual coolant.

Let us first briefly recall how the classical internal combustion (i.e., the Otto-cycle) engine works [9]. Figure 1 depicts a classical Otto-cycle engine in which a working gas passes through the cycle 1-2-3-4. In order to prepare for the next step, where we are going to take advantage of the internal degree of freedom of the engine fluid, i.e., a gas consisting of multilevel atoms or molecules, we consider the setup of an Otto-cycle engine consisting of two cylinders. This will allow us to introduce the high- $Q$ microwave cavity and laser systems in the setup in a natural way. This two-cylinder setup follows the classical stages of the Otto cycle. In the first stage 1-2 [Fig. 1(a)], the gas expands isotropically (i.e., no heat in or out) from volume $V_{1}$ to volume $V_{2}$ (here and below, $T, V, P$, and $S$ stand for temperature, volume, pressure, and entropy, respectively), doing useful (good) work given by $W_{g}=C_{v}\left(T_{1}-T_{2}\right)$, where $T_{2}=T_{1} / \mathcal{R}, C_{v}$ is the heat capacity, $\mathcal{R}=\left(V_{2} / V_{1}\right)^{\gamma-1}$, and $\gamma$ is the ratio of heat capacities at constant pressure to constant volume. Then, in the next stage 2-3 [Fig. 1(b)], we consider gas transfer from one cylinder to another and an isochoric cooling process (i.e., at constant volume). Let us underline that transferring gas from one cylinder to another plays no role in the classical Otto cycle, but we include these two cylinders here because it allows us to cool the internal degrees of freedom of the gas by using a high- $Q$ microwave cavity and a laser system, thus producing additional useful work. Heat $q_{\text {out }}$ is extracted isochorically by a heat exchanger (cooler). This happens at constant volume $\left(V_{2}=V_{3}\right)$, bringing the temperature down from $T_{2}$ to $T_{3}$. The pressure drops from $P_{2}=\left(R / V_{3}\right) T_{2}$ to $P_{3}$ $=\left(R / V_{3}\right)\left(T_{2}-q_{\text {out }} / C_{v}\right)$, where $R=N k$, where $N$ is the number of atoms and $k$ is Boltzmann's constant. At stage 3-4 [Fig. 1(c)], the gas is compressed isotropically from volume $V_{3}$ to volume $V_{4}$ requiring "waste" work, and the pressure becomes $P_{4}=P_{1} \mathcal{R}^{\gamma /(\gamma-1)}$. At stage 4-1 [Fig. 1(d)], heat $q_{\text {in }}$ is added isochorically when the piston is at the top dead center and brings the gas from state $\left(T_{4}, V_{4}, P_{4}\right.$, and $\left.S_{4}\right)$ to state $\left(T_{1}, V_{1}=V_{4}, P_{1}\right.$, and $\left.S_{1}\right)$. Note that this isochoric process brings the gas back to the starting state $\left(P_{1}, V_{1}, T_{1}\right)$, and ready to start a new cycle.

Now comes the key step. What if we replace the cooler 


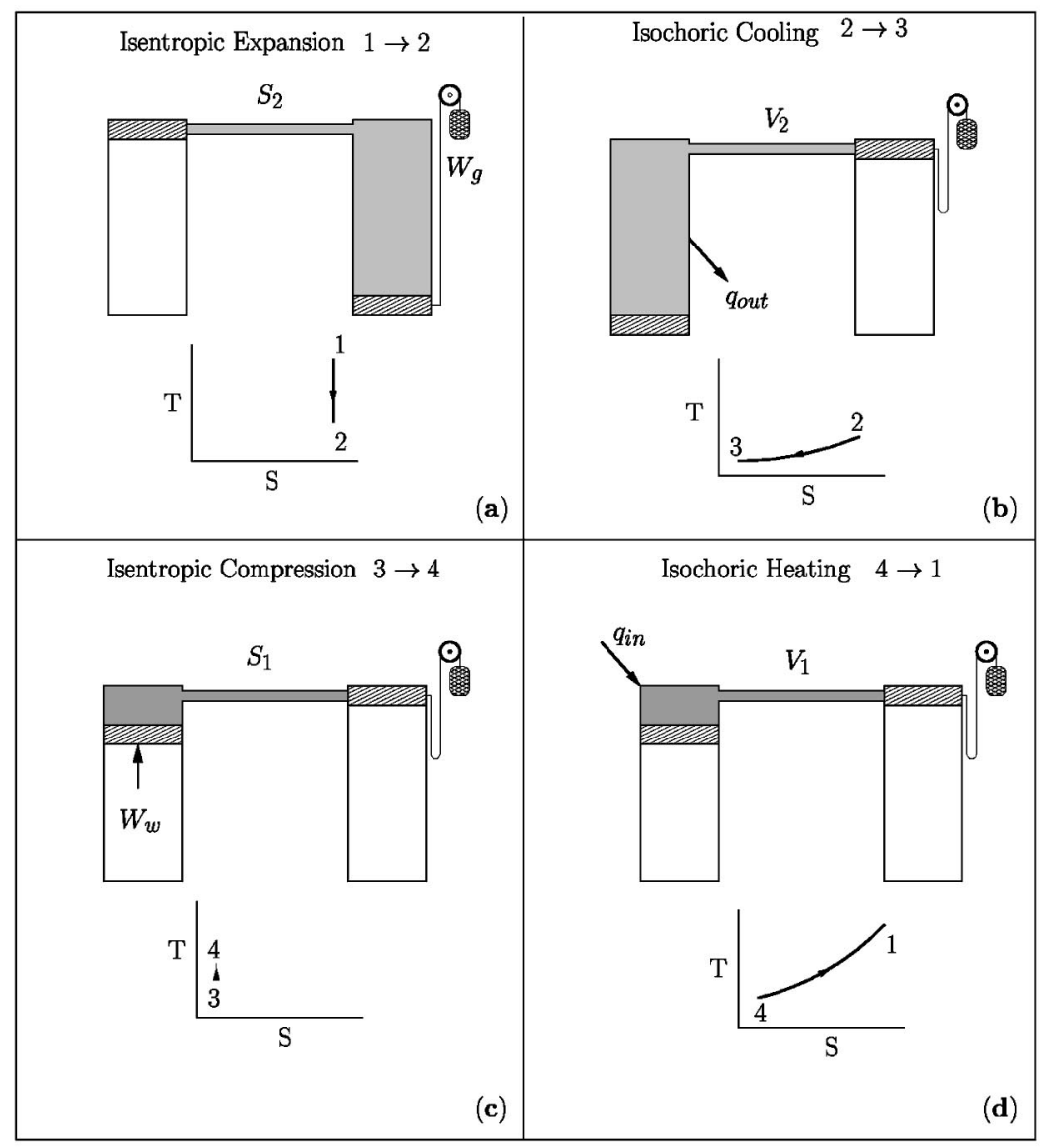

FIG. 1. Classical Otto cycle.

with a high- $Q$ microwave cavity and a laser system that could extract thermal energy stored in the internal degrees of freedom when taking us from thermodynamic states 2 to 3 ? Such an engine can properly be called a quantum engine and could, in principle, operate beyond the classical limit. Indeed it does, since $q_{\text {out }}$ is now partially converted into the emitted laser energy $W_{l}$ that can do useful work (see Fig. 2). In the following, we discuss a possible scheme for such a quantum Otto engine.

We consider the operating gas to consist of three-level atoms as shown in Fig. 3. The internal atomic states are chosen to be very long lived when the atoms are in free

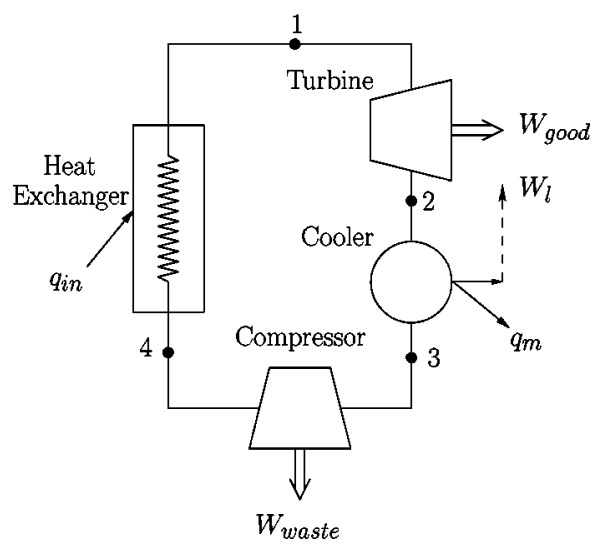

FIG. 2. General scheme of quantum Otto-cycle engine. space. However, the atoms are strongly coupled to the radiation field due to the increased density of states of the radiation field inside the high- $Q$ microwave and laser cavities. The high- $Q$ microwave cavity is resonant with the $|b\rangle \leftrightarrow|c\rangle$ transition and the laser cavity is resonant with the $|a\rangle \leftrightarrow|b\rangle$ transition.

The role of the microwave cavity at temperature $T_{3}$ is to cool the internal state $|b\rangle$ such that the population from level $|b\rangle$ is depleted to level $|c\rangle$. The cavity is tuned at the resonant frequency corresponding to the atomic transition between levels $|b\rangle$ and $|c\rangle$ (see Fig. 4). This resonant coupling between atoms and a thermal reservoir at temperature $T_{3}$ is much stronger than the off-resonant coupling at the $|a\rangle \leftrightarrow|b\rangle$ transition. Thus, the population relaxation rate for levels $|b\rangle$ and $|c\rangle$ is much faster than for levels $|a\rangle$ and $|b\rangle$ (but eventually all populations at all levels should be at thermal equilibrium at temperature $T_{3}$ ). Adjusting a proper interaction time inside the cavity, we can obtain that the populations in levels $|b\rangle$ and $|c\rangle$ are in thermal equilibrium at temperature $T_{3}$, but the population in level $|a\rangle$ remains untouched (its change is negligible). The heat extracted in this process is $q_{m}$.

The atoms then enter a laser cavity and are coherently driven from excited state $|a\rangle$ to ground state $|b\rangle$ by stimulated emission. This results in the coherent buildup of the laser field in the cavity and the emission of energy $W_{l}$, as indicated in Figs. 3 and 5. The cold atoms in state $|b\rangle$ then undergo collisional thermalization, cooling the center-of- 


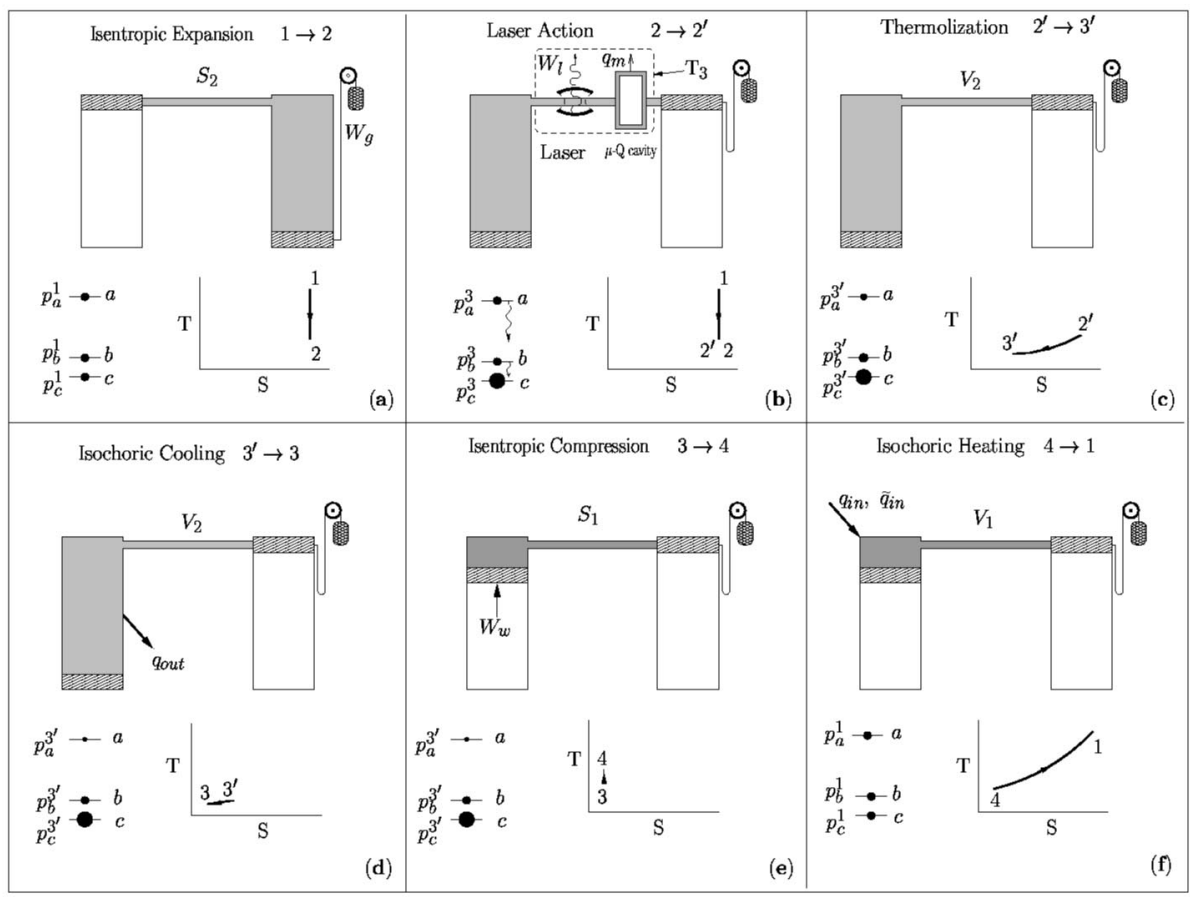

FIG. 3. The quantum Otto engine that incorporates the high- $Q$ microwave cavity and the laser system follows the cycle comprising the following steps: (a) $(1 \rightarrow 2)$ The gas expands isotropically (i.e., no heat in or out), yielding $W_{g}=C_{v}\left(T_{1}-T_{2}\right)$, where $T_{2}=T_{1} / \mathcal{R}$ as before. (b) $\left(2 \rightarrow 2^{\prime}\right)$ High- $Q$ microwave cavity and laser action. The high- $Q$ microwave cavity coupled to the transition $|b\rangle-|c\rangle$ depletes the $|b\rangle$ states, amounting to extraction of heat $q_{m}$. The laser action involves stimulated emission of energy $W_{l}$. Both high- $Q$ cavity and laser are at temperature $T_{3}$; (c) $\left(2^{\prime} \rightarrow 3^{\prime}\right)$ Thermalization of the gas to temperature $T_{3}^{\prime}>T_{3}$. (d) $\left(3^{\prime} \rightarrow 3\right)$ Isochoric cooling of external degrees of freedom of gas to temperature $T_{3}$. (e) $(3 \rightarrow 4)$ The gas is then compressed isotropically to volume $V_{4}=V_{1}$, requiring waste work $W_{w}$ $=C_{v}\left(T_{4}-T_{3}\right)$, where $T_{4}=T_{3} \mathcal{R}$. (f) $(4 \rightarrow 1)$ The gas is put in contact with a heat exchanger to bring the gas to the starting state $\left(P_{1}, V_{1}, T_{1}\right)$. During the heating, the external degrees of freedom absorb heat $q_{i n}=C_{v}\left(T_{1}-T_{4}\right)$, and internal degrees absorb $\tilde{q}_{i n}=q_{m}+W_{l}$. In the passage $2 \rightarrow 2^{\prime}$, laser radiation is extracted from the thermal distribution by depleting the lower $|b\rangle$ state populations, much as in lasing without inversion (LWI).

mass motion. Thus the "quantum heat exchanger" allows one to practically convert the heat out of the system, $q_{\text {out }}$, by heat out of a high- $Q$ microwave cavity, $q_{m}$, combined with useful work by the laser $W_{l}$. The specific entropy difference of the internal states between points 2 and 3 of Figs. 3 and 5 is easily calculated, as discussed below.

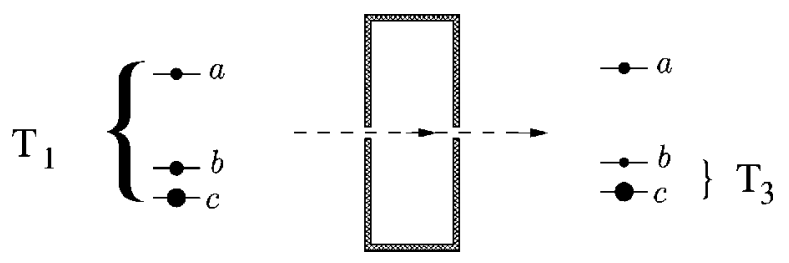

FIG. 4. Redistribution of the atomic populations in levels $|b\rangle$ and $|c\rangle$ after passing through a high- $Q$ microwave cavity. The cavity at temperature $T_{3}$ is tuned at the resonant frequency corresponding to the atomic transition between levels $|b\rangle$ and $|c\rangle$. This resonant coupling between atoms and a thermal reservoir at temperature $T_{3}$ is much stronger than the off-resonant coupling at the $|a\rangle \leftrightarrow|b\rangle$ transition. Thus, the atom having distribution of population corresponding to temperature $T_{1}$ before entering the cavity has the populations in levels $|b\rangle$ and $|c\rangle$ to be in a thermal equilibrium at temperature $T_{3}$ after the high- $Q$ microwave cavity, and the population in level $|a\rangle$ remains unchanged.
The laser energy is coherent useful work, whereas the high- $Q$ microwave cavity allows one to dump incoherent heat energy. This distinction is easily understood if we simply recall the thermal photon distribution functions inside the high- $Q$ microwave and laser cavities. The field density matrix inside the high- $Q$ microwave cavity is given by

$$
r_{n n}^{m}=\bar{n}_{m}^{n} /\left(\bar{n}_{m}+1\right)^{n+1},
$$

where $\bar{n}_{m}=1 /\left[\exp \left(\hbar \nu_{m} / k T_{3}\right)-1\right], \hbar \nu_{m}$ is the energy per quantum of the microwave field. The density matrix describing the laser field proceeds from an initial thermal state, which is largest for small $\bar{n}_{l}$, to the sharply peaked coherent distribution. For atoms passing through the cavity at a rate $r_{a}$, the photon statistics of the cavity radiation field $r_{n, n}$ is governed by the equation

$$
\begin{aligned}
\dot{r}_{n, n}= & -r_{a} \sin ^{2}(g \tau \sqrt{n}) r_{n, n}+r_{a} \sin ^{2}(g \tau \sqrt{n+1}) r_{n-1, n-1} \\
& -C\left[\bar{n}_{t h}(2 n+1)+n\right] r_{n, n}+C \bar{n}_{t h} n r_{n-1, n-1} \\
& +C\left(\bar{n}_{t h}+1\right)(n+1) r_{n+1, n+1}
\end{aligned}
$$




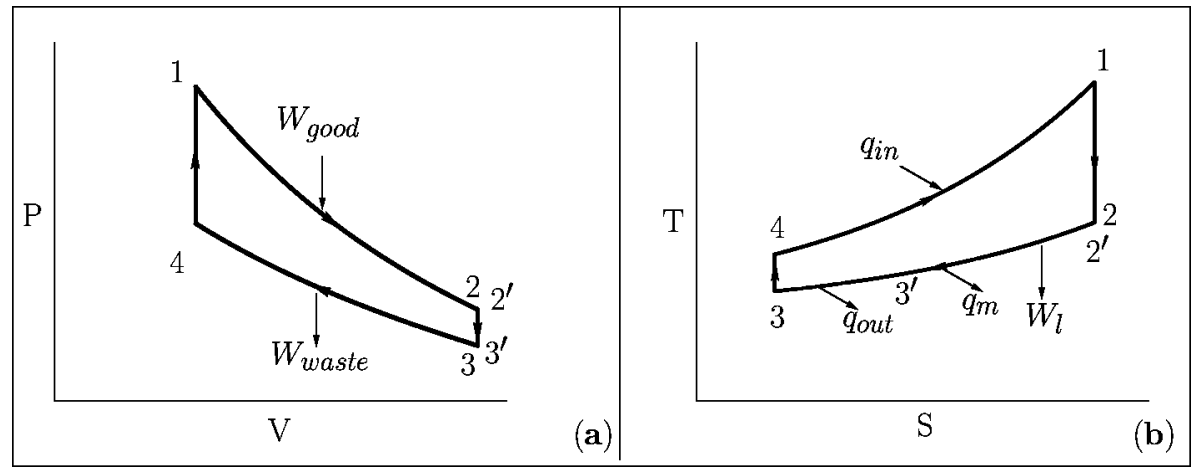

FIG. 5. Quantum Otto-engine thermal cycle. where $g$ is the atom-field coupling constant, $\tau$ is the interaction time of the atom with the cavity field, $C$ is the cavity loss parameter, and $\bar{n}_{t h}$ is the average number of thermal photons in the cavity at temperature $T_{3}$ with no atoms present. The interaction time $\tau$ is not fixed and depends on the velocity $v$ of atoms via $\tau=L / v$, where $L$ is the length of the cavity. We assume that the interaction time is much shorter than all decay rates in the system to justify the absence of absorption terms in Eq. (2). The velocity is given by a Maxwell-Boltzmann distribution and therefore we need to average Eq. (2) over the velocity distribution. For interaction times that are short compared to the Rabi frequency, i.e., $\tau n g \ll 1$, we can expand the sine functions in Eq. (2) and retain only the linear and the saturation terms. Thus, for the laser, Eq. (2) results in

$$
\begin{aligned}
\dot{r}_{n, n}^{(l)}= & -\left[A(n+1)-B(n+1)^{2}\right] r_{n, n}^{(l)}+\left[A n-B n^{2}\right] r_{n-1, n-1}^{(l)} \\
& -C\left[\bar{n}_{t h}(2 n+1)+n\right] r_{n, n}^{(l)}+C \bar{n}_{t h} n r_{n-1, n-1}^{(l)} \\
& +C\left(\bar{n}_{t h}+1\right)(n+1) r_{n+1, n+1}^{(l)}
\end{aligned}
$$

where $A=r_{a} g^{2}\left\langle\tau^{2}\right\rangle$ is the linear gain and $B=r_{a} g^{4}\left\langle\tau^{4}\right\rangle / 6$ is the nonlinear saturation parameter. Here angle brackets $\langle\ldots\rangle$ represent an average with respect to the MaxwellBoltzmann velocity distribution. A steady-state solution of Eq. (3) can be obtained via a detailed balance condition and is given by [10]

$$
r_{n, n}^{(l)}=r_{0,0} \prod_{l=1}^{n}\left[\frac{A-B l}{C\left(\bar{n}_{t h}+1\right)}+\frac{\bar{n}_{t h}}{\bar{n}_{t h}+1}\right] .
$$

This distribution function for photons is peaked at $(A$ $-C) / B$.

Having set the stage, we now sketch the thermostatistical analysis and calculate $W_{l}$ and $q_{m}$ microscopically. A complete rigorous calculation requires a quantum theory of the laser-type analysis. However, it is sufficient for the present purposes to apply microscopic energy balance conditions to obtain expressions for the important quantities.

We assume that atoms bounce many times back and forth through the microwave and laser cavities while the gas is moving adiabatically from right to left. Inside the high- $Q$ microwave cavity, the distribution of populations in levels $|b\rangle$ and $|c\rangle$ is established in accordance with temperature $T_{3}$; then, the population inversion between levels $|a\rangle$ and $|b\rangle$ can occur if temperature $T_{3}$ is low enough, and this leads to lasing that eliminates population difference between levels $|a\rangle$ and $|b\rangle$. After many bounces, the atoms settle down into the mixed steady state with equal populations in levels $|a\rangle$ and $|b\rangle$, i.e.,

$$
\rho_{\text {many }}\left(2^{\prime}\right)=p_{b}^{3} \Lambda_{a}+p_{b}^{3} \Lambda_{b}+\left(1-2 p_{b}^{3}\right) \Lambda_{c},
$$

where $\Lambda_{\alpha}=|\alpha\rangle\langle\alpha|, \alpha=\mathrm{a}, \mathrm{b}$, and c. However, it is necessary to mention here that at temperature $T_{3}$ (because of atomic interaction with the thermal field of the high- $Q$ microwave cavity), $p_{\alpha}^{3}$ is the probability of the atom being in level $\alpha$, and $p_{\alpha}^{3}$ is not a thermalized state, and, therefore, does not obey the Boltzmann distribution.

Next, we find an expression for population $p_{b}^{3}$. As we noted above, levels $|b\rangle$ and $|c\rangle$ are in thermal equilibrium at temperature $T_{3}$ after passage through the microwave cavity. Thus, it follows from Eq. (5) that

$$
\frac{p_{b}^{3}}{p_{c}^{3}} \equiv \frac{p_{b}^{3}}{1-2 p_{b}^{3}}=e^{-\epsilon_{b c} / k T_{3}} .
$$

This gives

$$
p_{b}^{3}=\frac{e^{-\epsilon_{b c} / k T_{3}}}{1+2 e^{-\epsilon_{b c} / k T_{3}}} .
$$

However, it is instructive to consider another derivation based on evolution to the steady state via atomic motion through the high- $Q$ microwave cavity and a laser system. The condition for the steady state is that after $m-1 \gg 1$ interactions with the laser system and high- $Q$ microwave cavity, the initial and final states of the atom do not change for any subsequent cycle. Thus, if an initial state for the $m$ th cycle is determined by populations $p_{b}^{3}$ and $p_{c}^{3}$, the final state is determined by populations $\left[p_{b}^{3}+\kappa\left(p_{b}^{3}+p_{c}^{3}\right)\right] / 2$ and (1 $-\kappa)\left(p_{b}^{3}+p_{c}^{3}\right)$ (see Fig. 6). The weight parameter $\kappa$ is determined by the Boltzmann distribution

$$
\frac{\kappa}{1-\kappa}=e^{-\epsilon_{b c} / k T_{3}}
$$

The steady-state conditions are

$$
p_{b}^{3}=\frac{1}{2}\left[p_{b}^{3}+\kappa\left(p_{b}^{3}+p_{c}^{3}\right)\right]
$$




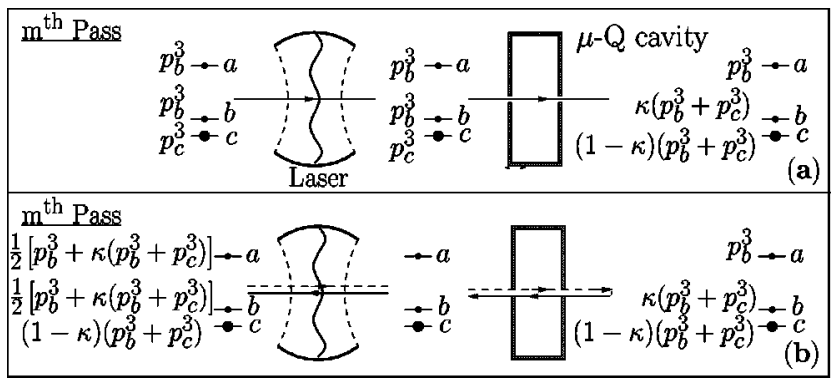

FIG. 6. Transformation of the atomic populations after passage through laser and high- $Q$ microwave cavity cooling system $m$ th time.

and

$$
p_{c}^{3}=(1-\kappa)\left(p_{b}^{3}+p_{c}^{3}\right)
$$

Conditions (9) and (10) are fulfilled when

$$
\kappa p_{c}^{3}=(1-\kappa) p_{b}^{3}
$$

Taking a normalization condition $p_{c}^{3}+2 p_{b}^{3}=1$ into account and solving Eqs. (8) and (11), we arrive again at Eq. (7).

We calculate $q_{m}$ by noting that $\left(p_{a}^{1}-p_{b}^{3}\right) N$ atoms go from $a$ to $b$, and $\left(p_{b}^{1}-p_{b}^{3}\right) N$ atoms go from $b$ to $c$. Thus, on making the $b \rightarrow c$ transition, the total incoherent energy added to the high- $Q$ microwave cavity field by all $N$ atoms is

$$
q_{m}=\epsilon_{b c} N\left[p_{a}^{1}-p_{b}^{3}+p_{b}^{1}-p_{b}^{3}\right] .
$$

Here, $p_{\alpha}^{i}\left(i=1\right.$ or $\left.3^{\prime}\right)$ is the probability of the atom being in level $\alpha$ at temperature $T_{i}$, i.e., $p_{\alpha}^{i}=Z_{i}^{-1} \exp \left(-\beta_{i} \epsilon_{\alpha}\right)$, where $\beta_{i}=1 / k T_{i}$ and $Z_{i}=\Sigma \exp \left(-\beta_{i} \epsilon_{\alpha}\right)$.

Likewise, $W_{l}$ is obtained by noting that the number of atoms going from $a$ to $b$ with the coherent emission of laser radiation is $N\left(p_{a}^{1}-p_{b}^{3}\right)$. Energy $\epsilon_{a c}-\epsilon_{b c}$ is given up by each atom, and the total coherent energy (i.e., useful work) given to the laser field is

$$
W_{l}=\left(\epsilon_{a c}-\epsilon_{b c}\right) N\left(p_{a}^{1}-p_{b}^{3}\right) .
$$

We may calculate the temperature following the laser cooler $T_{3}$, by noting that, for an ideal gas, any change in internal energy is strictly accounted for by temperature alone. Energy balance yields the following equation:

$$
C_{v} T_{2}+N \sum_{\alpha} \epsilon_{\alpha} p_{\alpha}^{1}=C_{v} T_{3^{\prime}}+N \sum_{\alpha} \epsilon_{\alpha} p_{\alpha}^{3^{\prime}}+W_{l}+q_{m},
$$

which leads to a transcendental equation given by

$$
T_{3^{\prime}}=T_{2}-N\left[\epsilon_{a c}\left(p_{a}^{3^{\prime}}-p_{b}^{3}\right)+\epsilon_{b c}\left(p_{b}^{3^{\prime}}-p_{b}^{3}\right)\right] / C_{v} .
$$

It is possible to show that inequality $T_{3^{\prime}} \geqslant T_{3}$ is always valid.

Now we calculate the efficiency of the quantum Otto engine, which is given by

$$
\eta_{q o}=\frac{W_{g}-W_{w}+W_{l}}{q_{i n}+q_{m}+W_{l}},
$$

where $W_{g}=C_{v}\left(T_{1}-T_{2}\right)$ is the work done during the gas expansion, and $W_{w}=C_{v}\left(T_{4}-T_{3}\right)$ is the waste work involved in the isotropic compression. We have $q_{\text {in }}=C_{v}\left(T_{1}-T_{4}\right)$, the heat taken in the isochoric process. We insert the values for $W_{g}, W_{w}$, and $q_{\text {in }}$ in Eq. (8) and use relations $T_{2}=T_{1} / \mathcal{R}$ and $T_{4}=T_{3} \mathcal{R}$ (since $V_{3}=V_{2}$ and $V_{4}=V_{1}$ ), where $\mathcal{R}$ $=\left(V_{2} / V_{1}\right)^{(\gamma-1)}$. The resulting expression for the quantum Otto-engine efficiency is

$$
\eta_{q o}=\eta_{o}+\delta \eta
$$

where

$$
\eta_{o}=1-\frac{1}{\mathcal{R}}
$$

is the efficiency of the classical Otto engine and

$$
\delta \eta=\frac{W_{l}+(1-\mathcal{R}) q_{m}}{\mathcal{R}\left[C_{v}\left(T_{1}-T_{4}\right)+W_{l}+q_{m}\right]}
$$

is the modification when the cooler is replaced by a high- $Q$ cavity and a laser system. The quantum efficiency of the Otto engine is improved for $\delta \eta>0$. This can happen for a wide variety of parameters.

For example, we can rewrite Eq. (17) in the form

$$
\eta_{q o}=\eta_{o}+\frac{W_{l}\left(1-\eta_{o}\right)-\eta_{o} q_{m}}{q_{i n}+W_{l}+q_{m}} .
$$

It follows from Eqs. (12) and (13) that

$$
\frac{W_{l}\left(1-\eta_{o}\right)}{q_{m} \eta_{o}}=\frac{\epsilon_{a b}}{\epsilon_{b c}}\left[\frac{p_{a}^{1}-p_{b}^{3}}{p_{a}^{1}-p_{b}^{3}+p_{b}^{1}-p_{b}^{3}}\right]\left[\frac{1-\eta_{o}}{\eta_{o}}\right] .
$$

We look for the condition when

$$
\frac{\epsilon_{a b}}{\epsilon_{b c}}\left[\frac{p_{a}^{1}-p_{b}^{3}}{p_{a}^{1}-p_{b}^{3}+p_{b}^{1}-p_{b}^{3}}\right]\left[\frac{1-\eta_{o}}{\eta_{o}}\right]>1
$$

We suppose that

$$
\zeta=\frac{\epsilon_{a b}}{\epsilon_{b c}}\left[\frac{1-\eta_{o}}{\eta_{o}}\right]>2 .
$$

Then,

$$
p_{b}^{3}<\frac{(\zeta-1) p_{a}^{1}-p_{b}^{1}}{\zeta-2}=\zeta^{\prime}
$$

Using Eq. (7), we finally get

$$
k T_{3}<\epsilon_{b c}\left[\ln \left(\frac{1-2 \zeta^{\prime}}{\zeta^{\prime}}\right)\right]^{-1} .
$$




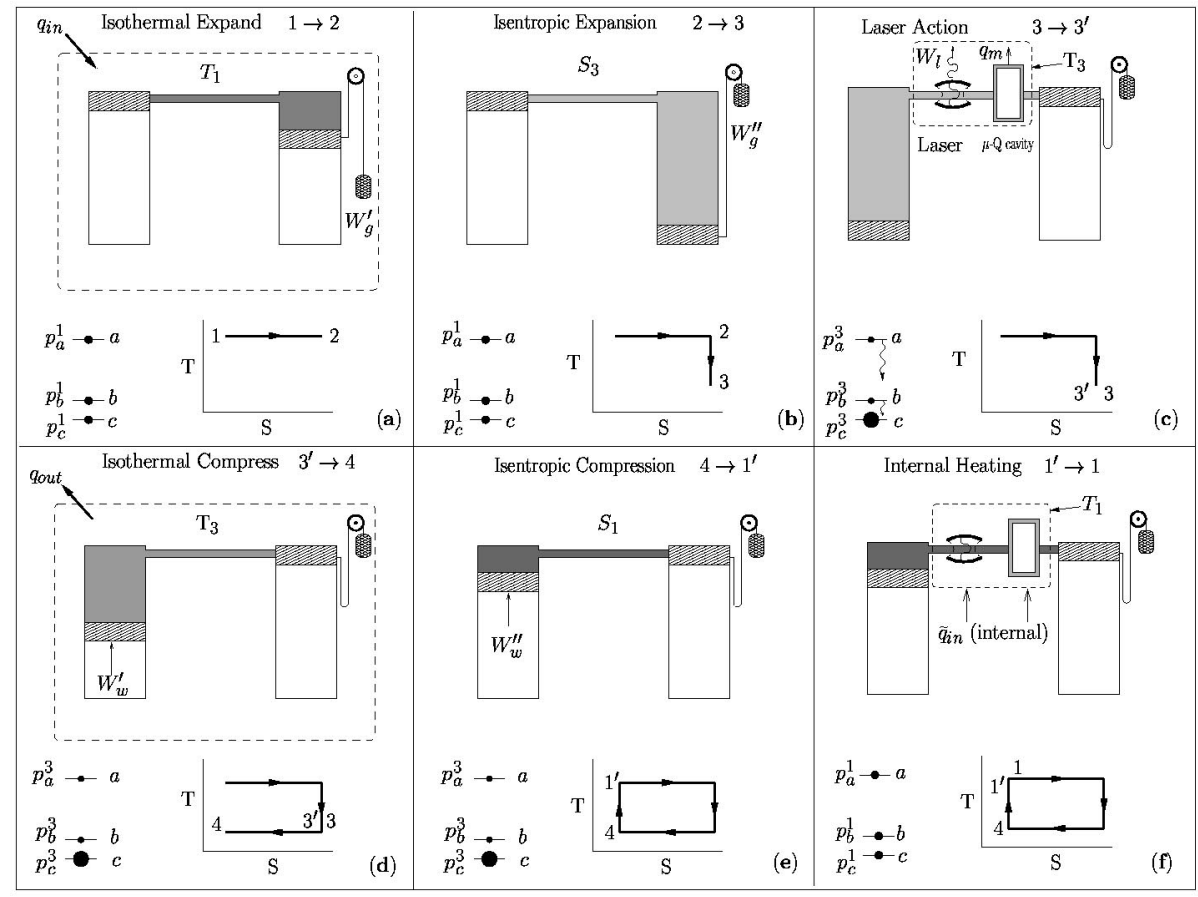

FIG. 7. The QCE operates as follows: (a) $(1 \rightarrow 2)$ A hot gas at temperature $T_{1}$ expands isothermally absorbing heat $q_{\text {in }}$ and doing good work $W_{g}^{\prime}$ raising a weight as indicated. (b) $\left(2 \rightarrow 3^{\prime}\right)$ The heat bath is removed and isotropically produces work $W_{g}^{\prime \prime}$. (c) $\left(3^{\prime} \rightarrow 3\right)$ Pistons move in conjunction so as to slowly move the gas of three-level atoms through the high- $Q$ microwave and laser cavities. Even though the atomic motion at point 3 is characterized by temperature $T_{3}$, we may arrange that the internal atomic degrees of freedom are effectively decoupled from the external center of mass motion. Thus the atom's internal population is still governed by temperature $T_{1}$, and upon passing through the cold laser (microwave) cavity, coherent (incoherent) radiation is emitted. (d) ( $3 \rightarrow 4$ ) The system is placed in a thermal heat bath, at $T_{3}$, and compressed to 4 ; this requires (waste) work $W_{w}^{\prime}$. Heat energy $q_{\text {out }}$ is removed in order to maintain temperature $T_{3}$. (e) $\left(4 \rightarrow 1^{\prime}\right)$ Gas is compressed isotropically with waste work $W_{w}^{\prime \prime}$. (f) $\left(1^{\prime} \rightarrow 1\right)$ Internal states are heated by hot cavities at temperature $T_{1}$ completing the cycle.

Thus, if we have a significantly low temperature $T_{3}$, we can improve the efficiency of the Otto engine. In order to show that it is possible to improve on the Otto cycle, we have performed numerical estimation. Using $\mathrm{CO}_{2}$ molecular gas under the conditions $T_{1}=1200 \mathrm{~K}, T_{2}=900 \mathrm{~K}$ and using liquid nitrogen as a coolant $T_{3}=77 \mathrm{~K}, T_{4}=200 \mathrm{~K}, V_{2} / V_{1}$ $=1.3$, the improvement in efficiency of the quantum Otto cycle over the classical Otto cycle is almost $25 \%$. Let us note that using a coolant at room temperature, $T_{3}=300 \mathrm{~K}$, decreases the efficiency improvement to $18 \%$, but it is still quite substantial.

\section{QUANTUM CARNOT ENGINE}

We have found that the efficiency of the quantum Otto engine is greater than that of the classical Otto engine. But what, if anything, can we learn from this, as concerns the second law? Is the efficiency of a Carnot-cycle engine improved by the same process of laser emission via hot exhaust atoms? This raises the interesting question of how the present work interfaces with the second law of thermodynamics; since it is well known that the engine efficiency exceeding that of the ideal Carnot cycle is tantamount to a violation of both the Clausius and Kelvin-Planck statements of the second law $[1,9]$. We, now consider a model for such a quantum Carnot engine (QCE).
In order to present the physics behind the envisioned QCE, consider Figs. 7 and 8 in which the working fluid passes through the cycle $1233^{\prime} 41^{\prime} 1$. In particular, we extend the classical Carnot engine to include a laser arrangement that can extract coherent laser energy from the internal degrees of freedom of the atoms at temperature $T_{1}$.

Note that, in Fig. 7(c), the energy dumped by the high- $Q$ microwave cavity is denoted by $q_{m}$. This emphasizes that it is incoherent heat energy that is being generated, because the thermal microwave radiation that brings the atom into a thermal distribution of populations between the $|b\rangle$ and $|c\rangle$ states contributes an irreversible evolution of the atom into its new configuration. This is in contrast to the buildup of the coherent laser field which is useful work. This is summarized in Fig. 8 where the usual $T-S$ diagram is extended to include the emission of laser energy $W_{l}$ and the high- $Q$ microwave cavity heat energy $q_{m}$ in the passage from 3 to $3^{\prime}$.

We turn now to the calculation of the efficiency of the QCE. As is shown in the following, we find

$$
\eta_{q c}=\eta_{c}+\frac{W_{l}\left(1-\eta_{c}\right)-\eta_{c} q_{m}}{W_{g}^{\prime}+W_{l}+q_{m}},
$$

where $\eta_{c}=1-T_{3} / T_{1}$ is the Carnot efficiency. We shall see 


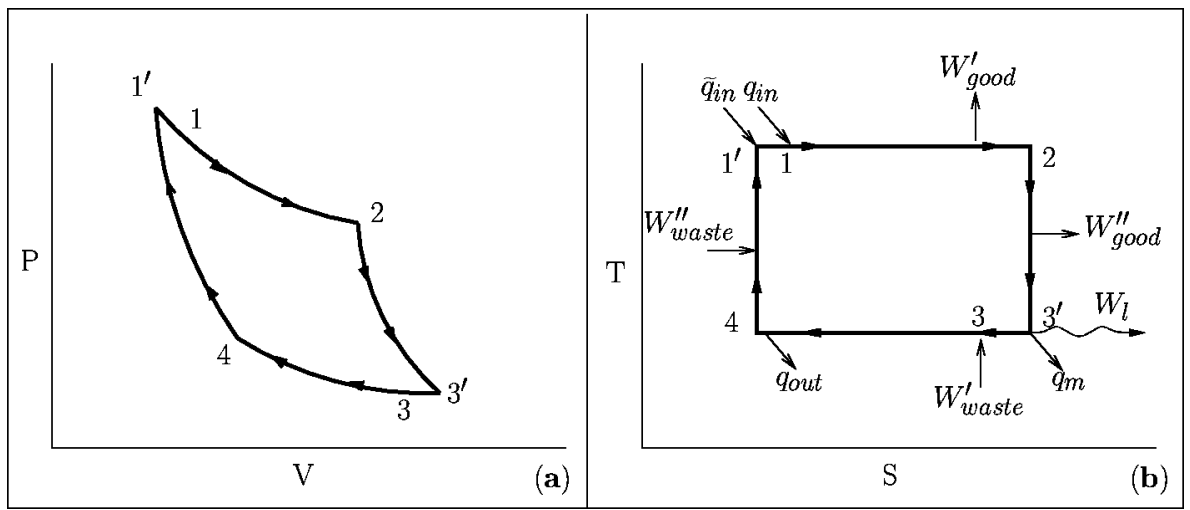

FIG. 8. Complete thermal cycle for QCE.

that $\eta_{q c}<\eta_{c}$, in agreement with the Carnot theorem that says that $\eta_{c}$ is the best we can hope for.

It is interesting to see how the second law is enforced here. To that end, we proceed with the analysis of the QCE. We seek the engine efficiency, defined as

$$
\eta_{q c} \equiv \frac{W_{g}^{\prime}-W_{w}^{\prime}+W_{l}}{q_{i n}+W_{l}+q_{m}}
$$

and/or

$$
\eta_{q c} \equiv \frac{q_{i n}+\tilde{q}_{i n}-q_{o u t}-q_{m}}{q_{i n}+W_{l}+q_{m}}
$$

where $W_{g}^{\prime}$ and $W_{w}^{\prime}$ are given by $N k T_{1} \ln \left(V_{2} / V_{1}\right)$ and $N k T_{3} \ln \left(V_{2} / V_{1}\right)$ respectively, and $N$ is the total number of the gas atoms in the engine. We will derive Eq. (26) by two different methods; the first is a physical thermodynamic approach, and the second a mathematical thermostatistical analysis.

The physical route to Eq. (26) through Eq. (27) is simple. The only unknown in Eq. (27) is $q_{\text {in }}$ and the only change from the classical Carnot engine is the extra internal energy $\tilde{q}_{\text {in }}$ added in the $1^{\prime} \rightarrow 1$ leg of the cycle. Work $W_{w}^{\prime \prime}$ is totally compensated by $W_{g}^{\prime \prime}$. Thus the energy out, $W_{g}^{\prime}, W_{l}$, and $q_{m}$, must come from $q_{i n}+\tilde{q}_{i n}$ in order to close the cycle, that is $q_{i n}+\tilde{q}_{i n}=W_{g}^{\prime}+W_{l}+q_{m}$, and inserting this into Eq. (27) yields Eq. (26).

The simple physical picture has appeal, but leaves unanswered many questions. For example, will the scheme really work? Atoms will bounce back and forth through the cavities many times. What does this do to lasing and relaxation due to the high- $Q$ microwave cavity? What are the actual expressions for $W_{l}$ and $q_{m}$ and what are the time scales involved? Where is the entropy going? Furthermore, it is interesting to see how the different pieces of a more complete mathematical analysis fit together to allow us to derive Eq. (26) from Eq. (28).

A careful analysis indicates that the calculation of $q_{m}$ and $W_{l}$ is carried out on the same lines as in the preceding section for quantum Otto engine and the resulting expressions are given by Eqs. (12) and (13), respectively.

The calculation of $q_{\text {out }}$ is simplified by our choice of long-lived atoms in which the internal and external degrees of freedom are decoupled. Thus, the internal states are just spectator states during the $3 \rightarrow 4$ (and $4 \rightarrow 1^{\prime}$ ) compressions, so

$$
q_{\text {out }}=N k T_{3} \ln \frac{V_{2}}{V_{1}}=W_{w}^{\prime},
$$

just as in the usual Carnot engine.

To calculate $q_{\text {in }}+\tilde{q}_{\text {in }}$, we note that it contains the energy $W_{g}^{\prime}$ needed to keep the gas at temperature $T_{1}$ during the 1 $\rightarrow 2$ expansion process as well as energy needed to bring the internal states to $T_{1}$ as in Fig. 7 . The heat $\tilde{q}_{i n}$ required to take the atoms from $\rho(1)$ to $\rho(3)$ is $N\left[\epsilon_{a c}\left(p_{a}^{1}-p_{b}^{3}\right)+\epsilon_{b c}\left(p_{b}^{1}\right.\right.$ $\left.\left.-p_{b}^{3}\right)\right]$. Hence the total heat intake is given by

$$
q_{i n}+\tilde{q}_{i n}=N k T_{1} \ln \frac{V_{2}}{V_{1}}+N\left[\epsilon_{a c}\left(p_{a}^{1}-p_{b}^{3}\right)+\epsilon_{b c}\left(p_{b}^{1}-p_{b}^{3}\right)\right] .
$$

We may write the second term in Eq. (30) as

$$
\begin{aligned}
& N\left[\epsilon_{a c}\left(p_{a}^{1}-p_{b}^{3}\right)-\epsilon_{b c}\left(p_{a}^{1}-p_{b}^{3}\right)+\epsilon_{b c}\left(p_{a}^{1}-p_{b}^{3}+p_{b}^{1}-p_{b}^{3}\right)\right] \\
& \quad=W_{l}+q_{m},
\end{aligned}
$$

where we have used Eqs. (12) and (13). Substituting Eq. (31) into Eq. (30) yields the "ansahlaulich" result

$$
q_{i n}+\tilde{q}_{i n}=N k T_{1} \ln \frac{V_{2}}{V_{1}}+W_{l}+q_{m}=W_{g}^{\prime}+W_{l}+q_{m},
$$

which agrees with $q_{\text {in }}$ used in deriving Eq. (26) from Eq. (27). Finally, we may use Eq. (32) to write $q_{\text {in }}+\tilde{q}_{\text {in }}-q_{\text {out }}$ $-q_{m}=\left(W_{g}^{\prime}+W_{l}+q_{m}\right)-W_{w}^{\prime}-q_{m}$, and, then, we insert this into Eq. (28) to obtain Eq. (27) and therefore $\eta_{q c}$, as before.

We note that the von Neumann entropy per atom, $S=-k \operatorname{Tr} \rho \ln \rho$, results from the heating of the internal states

$$
S\left(1^{\prime} \rightarrow 1\right)=-k\left[\Sigma_{\alpha} p_{\alpha}^{1} \ln p_{\alpha}^{1}-2 p_{b}^{3} \ln p_{b}^{3}-p_{c}^{3} \ln p_{c}^{3}\right]
$$

where $p_{c}^{3}=1-2 p_{a}^{3}$. The entropy $S\left(1^{\prime} \rightarrow 1\right)$ is equal and opposite to that removed in the $3^{\prime} \rightarrow 3$ high- $Q$ cavity and a laser energy-entropy extraction process. This is, of course, the analog of the Carnot statement that $q_{\text {in }} / T_{1}=q_{\text {out }} / T_{3}$ for the external degrees of freedom.

From Eqs. (12) and (13) we have 


$$
\frac{W_{l}\left(1-\eta_{c}\right)}{q_{m} \eta_{c}}=\frac{\epsilon_{a b}}{\epsilon_{b c}}\left[\frac{p_{a}^{1}-p_{b}^{3}}{p_{a}^{1}-p_{b}^{3}+p_{b}^{1}-p_{b}^{3}}\right]\left[\frac{1-\eta_{c}}{\eta_{c}}\right],
$$

and, since $p_{a}^{1} \lesssim p_{b}^{1}$ for a hot gas, we may write

$$
\frac{W_{l}\left(1-\eta_{c}\right)}{q_{m} \eta_{c}} \lesssim \frac{\epsilon_{a b}}{\epsilon_{b c}}\left[\frac{1-\eta_{c}}{\eta_{c}}\right]=\frac{\epsilon_{a c}-\epsilon_{b c}}{\epsilon_{b c}}\left[\frac{1-\eta_{c}}{\eta_{c}}\right] .
$$

But, for lasing, we require

$$
p_{a}^{1}=\frac{\exp \left(-\frac{\epsilon_{a c}}{k T_{1}}\right)}{Z_{1}}>p_{b}^{3}=\frac{\exp \left(-\frac{\epsilon_{b c}}{k T_{3}}\right)}{1+2 \exp \left(-\frac{\epsilon_{b c}}{k T_{3}}\right)},
$$

which is equivalent to $\epsilon_{a c} / k T_{1}<\epsilon_{b c} / k T_{3}$ and we may write

$$
\frac{\epsilon_{a c}}{\epsilon_{b c}}<\frac{T_{1}}{T_{3}} .
$$

Inserting $\eta_{c}=\left(T_{1}-T_{3}\right) / T_{1}$ and Eq. (36) into Eq. (35) we find

$$
\frac{W_{l}\left(1-\eta_{c}\right)}{q_{m} \eta_{c}}<1
$$

Hence $\eta_{q c}<\eta_{c}$, as noted earlier.

\section{CONCLUSION}

Classical thermodynamics deals with classical, largely ideal, gases often ignoring internal degrees of freedom. This yields certain limits on the efficiency of heat engines involving such ideal gases. However, real gases also have internal quantum structure, which, once properly taken into account, can allow one to improve some aspects of heat engine operation. In the present paper it is suggested that additional useful work could be extracted in the form of coherent laser radiation.

In particular, we have shown that the classical Otto heat engine can be improved by an idealized quantum high- $Q$ microwave cavity and a laser system enabling us to extract useful work from the internal degrees of freedom of a working three-level gas. We have shown that a classical Carnot heat engine cannot be improved in this way. The present analysis focuses on a simple model of engine behavior. Future work will be directed toward more practical systems and laboratory demonstrations.

\section{ACKNOWLEDGMENTS}

The authors gratefully acknowledge the support from the DARPA, the Office of Naval Research, and the Texas Advanced Technology and Research Programs.
[1] For an insightful discussion of the physics see E. Lieb and J. Yngvason, Phys. Today 53(10), 12 (2000); for applications see K. Wark, Advanced Thermodynamics for Engineers (McGrawHill, New York, 1995).

[2] A. Allahverdyan and Th. Nieuwenhuizen, Phys. Rev. Lett. 85, 1799 (2000); See also P. Weiss, Sci. News (Washington, D.C.) 158, 225 (2000).

[3] The first law is stated physically and mathematically as "There are no perpetual motion machines of the first kind" and "The change in internal energy is an exact derivative which has to be zero for any isolated system." The second law is stated in many different ways, for example, in a textbook we read: [T]he Kelvin-Planck statement of the second law of thermodynamics is expressed as follows: "It is impossible for any device that operates on a cycle to receive heat from a single reservoir and produce a net amount of work." In this context, Wark says: "A device which violates the above statement is known as a perpetual-motion machine of the second kind. It produces work solely by the cooling of a body. Hence we cannot directly use the vast quantities of energy in the oceans and the atmosphere on earth to produce work." The change in entropy is an exact derivative which cannot be negative for any isolated system. In the words of Planck: "The Second Law of Thermodynamics, with all its extensions, has become the Principle of Increase of Entropy . ...."

[4] M.O. Scully, Phys. Rev. Lett. 87, 220601 (2001).

[5] D. Klepnner and S. Haroche, Phys. Today 42(1), 24 (1989); S. Haroche and J. M. Raimond, in Atomic and Molecular Physics edited by D. Bates and B. Bederson (Academic, New York, 1985), Vol. 20, p. 350; D. Meschede, H. Walther, and G. Müller, Phys. Rev. Lett. 54, 551 (1985); A review is given by G. Raithel, C. Wagner, H. Walther, L. M. Narducci, and M. Scully, in Cavity Quantum Electrodynamics, edited by P. R. Berman (Academic, Boston, 1994), p. 57; The first theory for the micromaser was given by P. Filipowicz, J. Javanainen, and P. Meystre, Phys. Rev. A 34, 3077 (1985).

[6] For reviews of current LWI studies see O. Kocharovskaya, Phys. Rep. 219, 175 (1992); M.O. Scully, ibid. 219, 191 (1992); S. Harris, Phys. Today 36(7), 36 (1997); J. Mompart and R. Corbalan, J. Opt B: Quantum Semiclassic. Opt. 2, R7 (2000). The first masers actually operated via LWI-type state selection, exactly as in Fig. 2(b), using a thermal beam. As such, it may be said that they were operating without inversion in a global sense. We have other examples of $P M_{3}$ operation closer to the current LWI research but they are not as simple and will be published elsewhere.

[7] O. Kocharovskaya, Y.V. Rostovtsev, and A. Imamoglu, Phys. Rev. A 58, 649 (1998).

[8] M.O. Scully, Phys. Rev. Lett. 88, 050602 (2002).

[9] F. Sears, Thermodynamics (Addison-Wesley, Massachusetts, 1959).

[10] The photon statistical distribution for the laser is found in M.O. Scully and W.E. Lamb, Phys. Rev. Lett. 16, 853 (1966); The derivation of M. Lax is to be found in W. Louisell, Quantum Statistical Properties of Radiation (Wiley, New York, 1973). 